\title{
Bending Behaviors of Azobenzene-containing Liquid Crystalline Polymers (AZ-LCP): Factors Influencing Bending Direction, Frequency and Degree
}

\author{
Yin junyi $1^{1, *}$ \\ ${ }^{1}$ Sichuan University, No.24 South Section 1, Yihuan Road, Chengdu , China
}

\begin{abstract}
Liquid crystal polymers (LCP) containing azobenzene and its derivatives are fascinating and topics of intense scientific curiosities. As the addition of photo-responsive azobenzene, the LCP is exerted with the photochemical response, which shows fascinating and useful photo-induced motions and consequently enables a variety of applications in a lot of fields. In this review, we detail the macroscale mechanical motion of azobenzene containing liquid crystalline polymer (AZ-LCP) materials upon irradiation, with emphasis on the external and internal factors of bending behaviors of AZ-LCP, including bending direction, frequency and degree. We end with an outlook of challenging and competitive application as soft actuators.
\end{abstract}

\section{Introduction}

It is well known that both azobenzene and liquid crystal are particularly attractive and remain a topic of intense scientific curiosity. The self-assembly performance of liquid crystals and the photo-responsive properties brought by the addition of azobenzene group make AZLCP materials capable of the photoinduced motions, and allows precise and remote controls spatially by multiple adjustability such as polarization and intensity. Spurred by these soft materials light-controllable properties with chosen suitable light, the applications become fascinating in various fields, which include a lot of photo-driven devices [1] like the photomechanical energy harvesting [2], miniaturized transport, artificial flytrap [3] and now even extending into area of nanotemplates for nanoengineering and nanofabrication [4].

Photo-actuator of AZ-LCP has made progress within these fifteen years, starting with research on the conditional light-induced reversible volume changes only within thickness ranging from 250 to $1400 \AA$ in 2005[5]. Then investigation gradually focus on the bending form, namely fast and large amplitude oscillation which is similar to hummingbird wingbeat and with little fatigue over cycles of AZ-LCP reported in 2008 [1]. In the following, the movement is converted and amplified into spring-like materials bending in twisting motions in 2014 [6], which displays a great qualitative improvement compared with the seemingly small motion than heretofore. Afterward, research in 2017 took advantage of fast cis-to-trans thermal relaxation to make the polymer films exhibit mechanical waves [2]. In the same year, Wani and his team designed a light-driven flytrap within the fiber-sized architecture [3]. In the previous reviews, researchers concluded various applications of AZ-LCP materials such as the strategy and progress of designing the soft robotics [7], and some mainly focus on LC or LCP like various systems of liquid crystal containing materials [8], the historical development of liquid crystalline polymeric materials [9] and recent findings about photo-responsive liquid crystalline block copolymers (PLCBCs) [10]. However, no one has ever analyzed the influence factors of the photoinduced bending behavior of the AZ-LCP materials upon irradiation until now, and it is of great importance to help us to do further research. The following review might perfectly full the blank in this research area and enable us to design the new soft actuator with controllable bending direction, frequency and degree that perfectly match our needs and explore the potential application in the emerging industry like micromechanical industry and artificial industry [6].

In this review, we discuss the remarkable properties of the macroscale photomechanical behaviors of AZ-LCP materials upon irradiation, and focus specifically on the factors that influence its bending properties. We start by briefly introducing four factors of the bending direction, including phase-state of azobenzene [11, 12], initial alignment of mesogens [13], location of the azobenzene [14-17] and the polarization direction of light[18]. Then current reported materials on the affect of the oscillating frequency such as frequency of the laser blocking, cantilever thickness [1], materials, order parameter [19] and substituents to the azobenzene moiety are reviewed [15, 20-22]. After this, we discussed the important roles played by the thermomechanical properties, the temperature $[23,24]$ and the angle between molecules and long axis of ribben [6] in bending degree. Based on

* Corresponding author: y2428799628@163.com 
the existent analysis, our researchers can already manipulate ideal motion through designing novel molecular structures to achieve complex motion. For example, we can adjust its photomechanical performances like making the bending angle larger, the photo-responsive speed faster and fabrication process of AZ-LCP materials further improved. Last but not least, we close with a forward-looking overview of this materials about its area for applications.

\section{Factors on bending directions}

The phase-state of AZ-LCP may have an effect on the photomechanical behaviors. Ikeda and his co-workers had reported photoinduced bending towards the light source when liquid crystalline polymer was applied. Figure 1(a) gives out the possible mechanism based on two preconditions: (1) the film has a large molar extinction coefficient, the incident UV light could only penetrate the surface layer ( $<1 \mathrm{um})$ of the film; (2) the volume of aligned mesogens would contract upon UV irradiation. However, Barrett et. al demonstrated quite different photodeformation behaviors with amorphous azobenzene-containing polymers in which the azobenzene groups are disordered. As shown in figure 1(b), the film expanded upon the incident light, and could partially recover as the incident light was withdrawn. The surface expansion effect might cause the film bending away from the light source. In comparison, the reversed bending directions of azobenzene containing films might be attributed to whether the film is composed of liquid crystalline polymer or amorphous polymer.
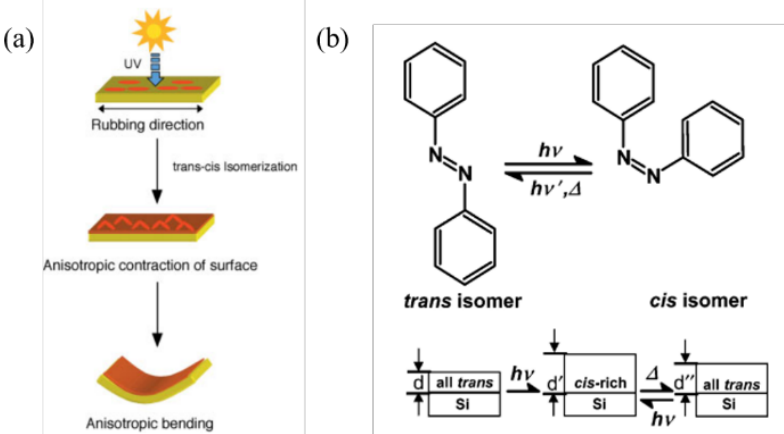

Fig. 1. (a) The mechanism of azobenzene-containing liquid crystalline polymer bending toward light source upon UV irradiation. Reproduced with permission from Ref.[2]; (b) Thickness change of amorphous azobenzene-containing polymer upon UV irradiation. Reproduced with permission from Ref. [3].

Mizuho Kondo and his team has discovered that photoinduced bending behavior of liquid-crystalline elastomer(LCE) was affected by initial alignment direction of mesogens within the film [13]. They investigated the bending behaviors of two alignment modes: homogeneous and hemeotropic. Figure 2(a) and 2(b) show the performance of the films under the irradiation of unpolarized UV light, which indicates that (1) the homeotropic film bent away from the UV light (2) the homogeneous film bends toward the UV light along the alignment direction of mesogens. Figure 3(c) indicated the mechanism of corresponding bending behaviors enlarged from the the trans-cis photoisomerization behaviors of azobenzene mesogens. In the case of homogeneously aligned films, the irradiated mesogens on the surface contracts upon UV irradiation, resulting in the bending towards light source; while when the mesogens are hemeotropically aligned, the surface conducted expansion and thus causing the film bend away from the light source. (a) 1)

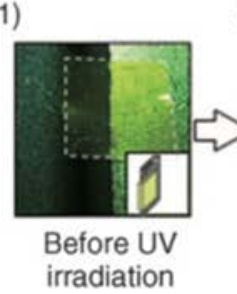

2)

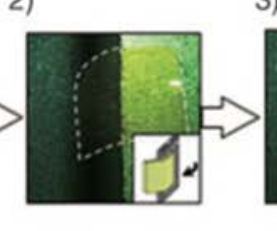

$366 \mathrm{~nm}, 3.1 \mathrm{~s}$
4)

3)
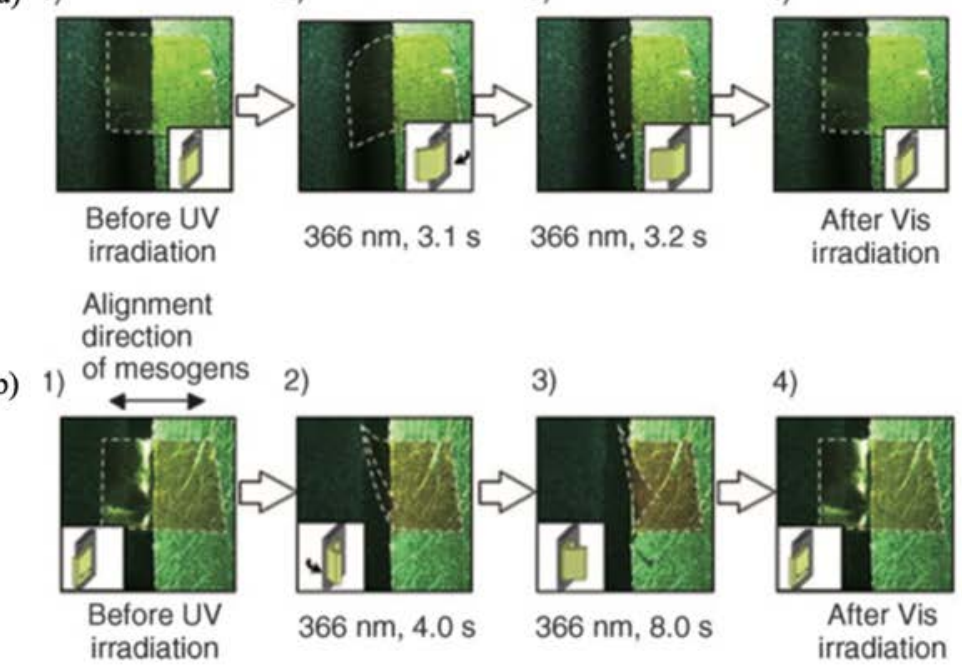

2)

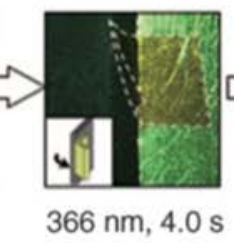

3)

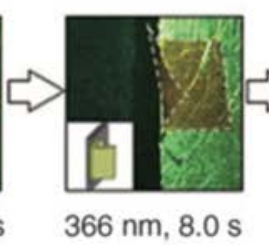

4)

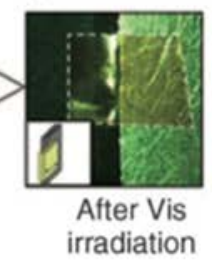

(c)

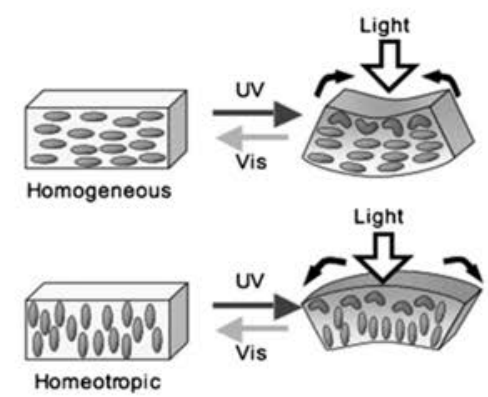

Fig. 2. (a) Bending behavior of the homogeneous film (b) and the homeotropic film. (c) Schematic illustration of the change. Reproduced with the permission from Ref. [11] 
The position of photo-responsive mesogens in crosslinked AZ-CLCP also contributes to the reversible changes. According to the revealed photoinduced contraction or expansion behavior [14, 16, 17, 25], Yu and his team detected that the film containing homogeneously-aligned AZs in side chain bends away from the light source above a specific temperature [26]. They put the AZ-CLCP fibers on a hot state and irradiated with UV light(365 nm), and it was observed to bend away from the light source when the temperature increased above $80{ }^{\circ} \mathrm{C}$. Meanwhile, in previous work [8, $12,13,27-29]$, the overall photomechanical response has a common feature: the CLCP films or fibers, which bend toward light source, have photoactive $A Z$ in the crosslinker. Therefore, they infer that whether $\mathrm{AZ}$ is located in the crosslinker or not plays an important role in deciding the bending direction of the CLCP material. Figure 3 displays the difference between them distinctly.
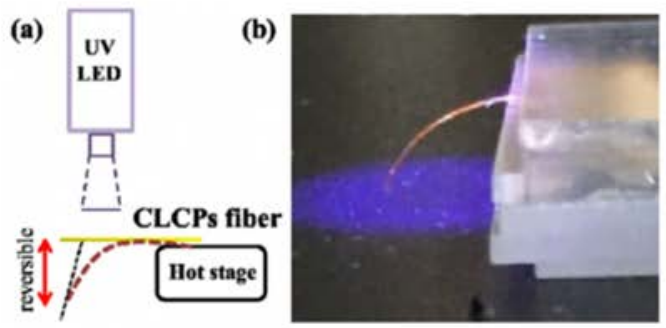

(c)
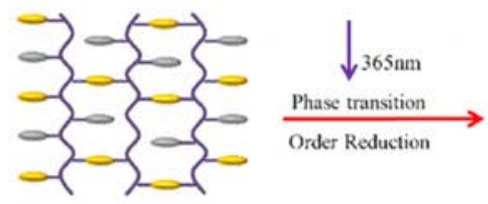

Azobenzene in crosslinker

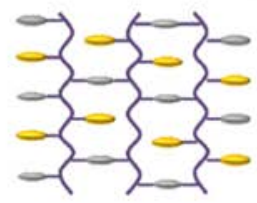

Azobenzene in side-chain

- Azobenzene

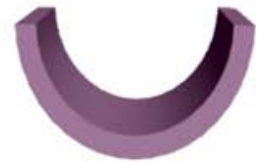

Toward UV light
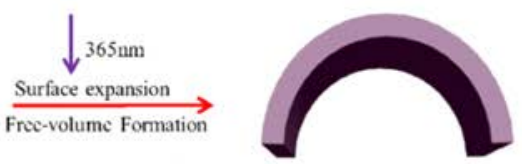

Away from UV light

$\triangle$ Non-photoactive mesogen

Fig. 3. (a) Experimental setup for photoinduced bending behavior of CLCP fibers upon irradiation with UV light. (b)significant bending of the fibers at temperature above $80 \mathrm{oC}$ (c)Schematic illustration of the different photoinduced deformation of AZ in crosslinker and side chain. Reproduced with the permission from Ref. [7]

The polarization direction of incident light also plays an important role in the photomechanical behaviors [18].Yu and coworkers prepared the films by polymerizing a liquid-crystal monomer (molecule 1 ) and a diacrylate crosslinker (molecule 2$)(9: 1, \mathrm{~mol} / \mathrm{mol})$, both of which possess azobenzene moieties as shown in figure 4a [18]. After altering the polarization direction of light at $366 \mathrm{~nm}$ to $45^{\circ}, 90^{\circ}$ and $135^{\circ}$ are shown in the fourth, sixth and eighth frames (figure $4 \mathrm{~b}$ ), respectively. It obviously indicates that the film is bent towards the incident light, and parallel to the polarization direction.

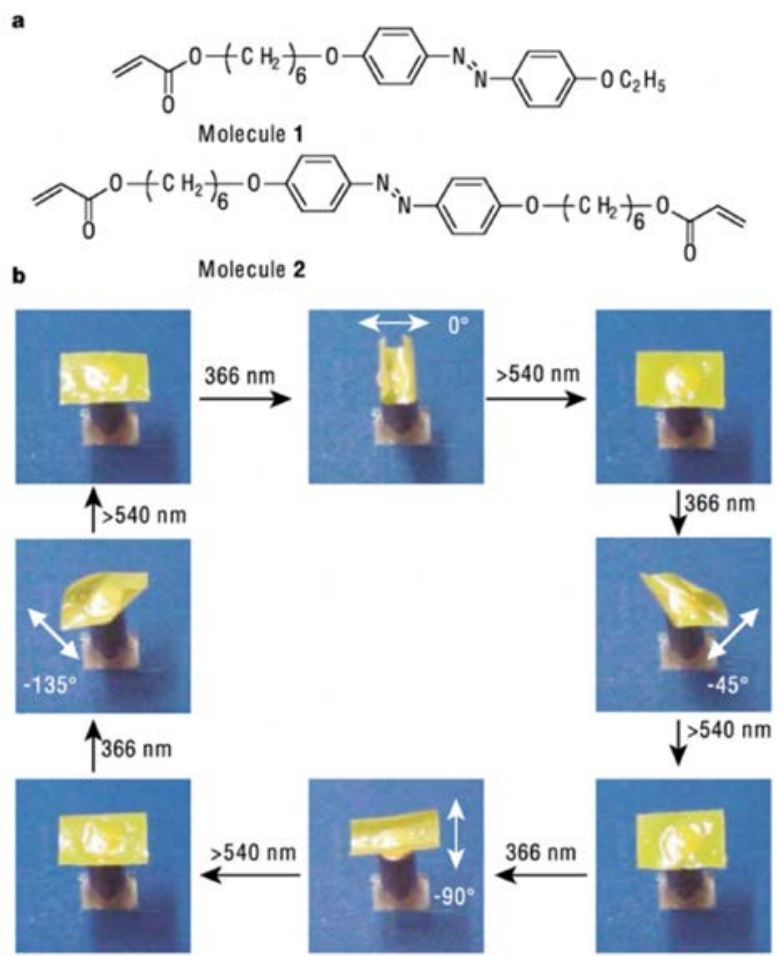

Fig. 4. (a) Chemical structure of the liquid-crystal monomer (molecule1) and crosslinker (molecule2). (b)The bending direction changes as the difference in angle of the linear polarized light. Reproduced with permission from Ref. [13]

\section{Bending frequency}


We summarized a variety of researches concentrated on the bending time and frequency of cantilever containing azo-LCN, and the related data of these materials is shown in Table 1.

Table 1. Oscillating Speed of Azobenzene liquid-crystalline polymers.

\begin{tabular}{|c|c|c|}
\hline material & $\begin{array}{l}\begin{array}{l}\text { frequency } \quad(\mathrm{Hz}) \\
\text { bending time(s) }\end{array} \\
\end{array}$ & reference \\
\hline azo-LCN & $10 \mathrm{~Hz}$ to almost $30 \mathrm{~Hz}$ & {$[1]$} \\
\hline PDLC & $20-40 s$ & [19] \\
\hline PD-20CL-5 & $\begin{array}{l}\text { a few seconds to many } \\
\text { minutes }\end{array}$ & [29] \\
\hline A6M6 & 200-2000s & \\
\hline AzoPy & $60-2500 \mathrm{~s}$ & \\
\hline I & $4-400 \mathrm{~s}$ & {$[2]$} \\
\hline II & $3-40 s$ & \\
\hline DR1A & $0.09-2 \mathrm{~s}$ & \\
\hline
\end{tabular}

Timothy J. White and his team reported the oscillation of the azo-LCN cantilever when exposed to $\mathrm{Ar}+$ laser beam. They discovered that the frequency of the oscillating depends on the frequency of the laser blocking and the cantilever thickness [1]. They discussed the oscillatory regularity of cantilevers with exposure to the laser beam. The optical setup displayed in figure $5 \mathrm{a}$ utilizes a spherical lens and a polarization rotator between the light source and azo-LCN cantilever to control the polarization direction of incident light. The rotation can be measured by the tip of cantilever with its displacement angle. When polarization parallel to the nematic director (long axis of the polymer cantilever), the cantilever bends from vertical (figure $5 \mathrm{~b}-\mathrm{i}$ ) to $42^{\circ}$ towards the laser source(E//n) (figure $5 b-i i)$. And then, when polarization perpendicular to $\mathrm{n}(\mathrm{E} \perp \mathrm{n})$, the azo-LCN bends nearly $90^{\circ}$ (figure $5 \mathrm{~b}$-iii). Once the cantilever is unexposed, it recover to vertical from either states (figure $5 b$-iv).

Besides, they tested the corresponding frequency of the oscillating azo-LCN cantilever through increasing the cantilever thickness. As figure 5c shows, when thickness of azo-LCN increase from $20 \mu \mathrm{m}$ to $50 \mu \mathrm{m}$, the amplitude rises from $10 \mathrm{~Hz}$ to almost $30 \mathrm{~Hz}$.

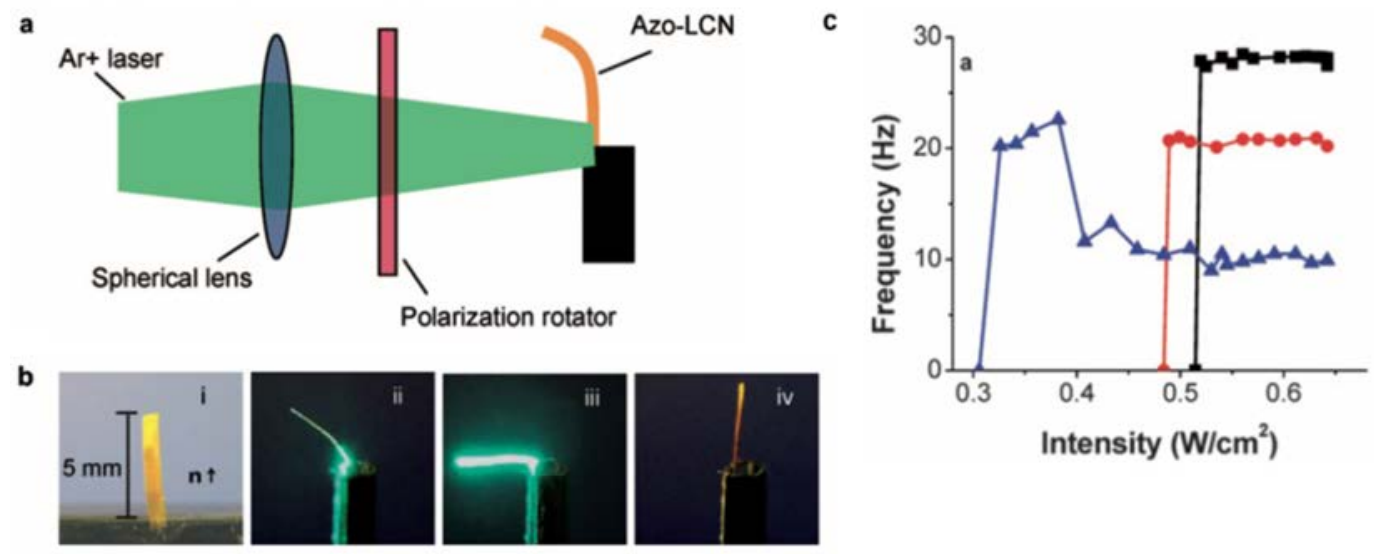

Fig. 5. (a) The setup made up of an Ar+ laser, a spherical lens, and a polarization rotator is utilized to make the azo-LCN cantilever photoactuated upon the laser. (b) The nematic director of the polymer is parallel to long axis of the polymer cantilever. In the beginning, the polymer cantilever is vertical (panel i). When polarization parallel to the long axis(E//n), the cantilever bends from vertical to 42 。 towards the laser source (panel ii). After that, when polarization perpendicular to $n(E \perp n)$, the azo-LCN bends nearly 90。 (panel iii), and without exposure, the cantilever recovers to vertical from either ii and iii states. (panel iv) (c) Frequency is faster with increased azo-LCNs thickness for $50 \mathrm{~mm}(\mathbf{I}), 40 \mathrm{~mm}(\mathbf{O})$, and $20 \mathrm{~mm}(\mathbf{\Delta})$ Reproduced with permission from Ref. [14]

$\mathrm{Yu}$ and his team synthesized a polymer dispersed liquid crystalline (PDLC) material based on the lightresponsive AZ mesogens and proved that both materials and order parameter play important roles in the bending frequency[19]. They made use of the phase reversion of PM6ABOC2 to obtain wrinkled LCP microparticles (LCPMs), and incorporate the microparticles into PVA solution (10\%) to fabricate the PDLC films. Furthermore, they mechanically stretched the film at a specific elongation rate (the ratio of the stretched to original samples) to make the deformation from spheres to ellipsoids (figure 6a), which can be tested and proved through Polarized Optical Microscope (figure 6b). Upon irradiation, the bending time of the PDLC film(about 20$40 \mathrm{~s}$ ) is relatively longer than the film made of LCN reported in Ref.[1] (figure 6c), which means that LCPM 
needs more time to recover to flat. Meanwhile, the bending time decreased with the increasing of elongation rate as demonstrated in figure $6 \mathrm{c}$.
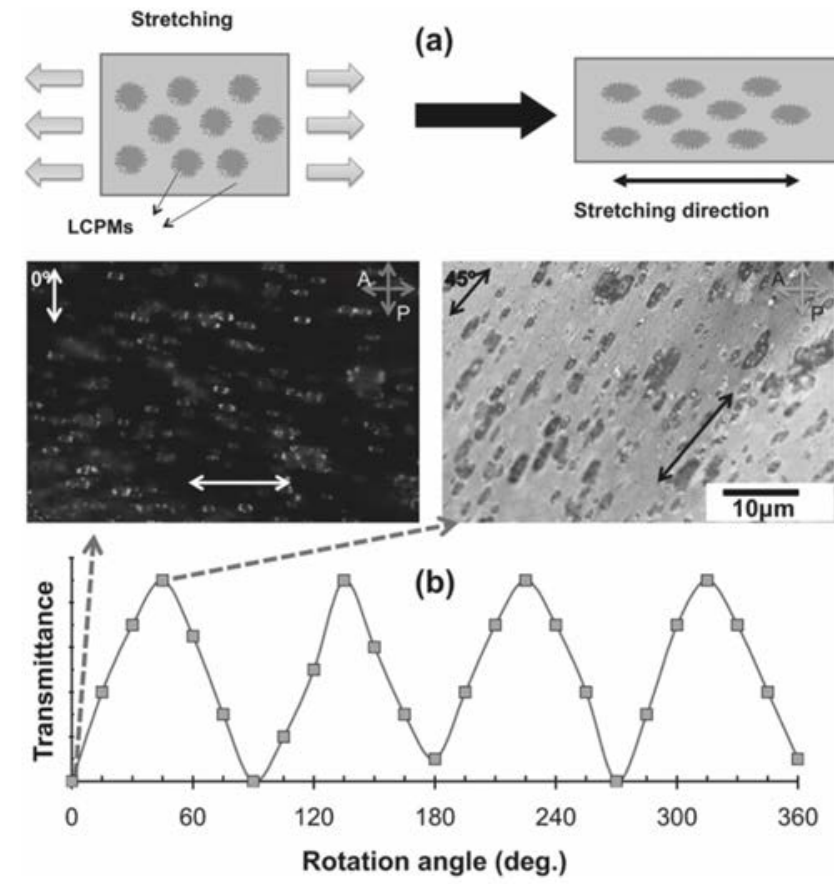

(a)
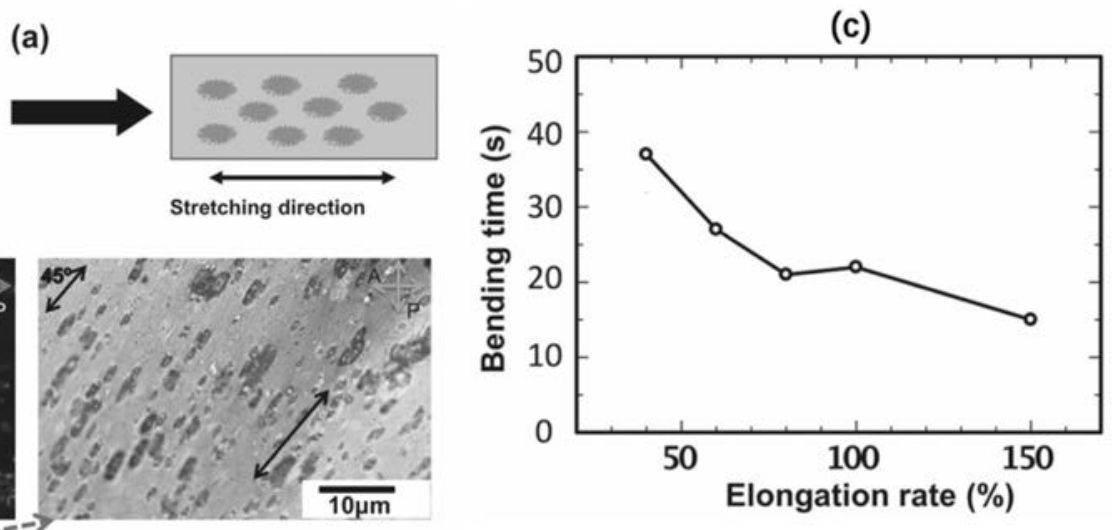

Fig. 6. (a) Schematic illustration of LCPM mechanical forming process and change of the LCPM photoinduced bending behaviors after mechanical stretching. a) Feasible scheme of LCPMs in the hybrid films. b) Polarized optical microscope images of hybrid LC films and transmittance of different rotation angle through two crossed polarizers (A, analyzer; P, polarizer). c) Relationship between elongation rate and bending time. Reproduced with permission from Ref. [15]

However, compared with the fast bending behaviors, it costs more time to regress after the photoisomerization. Anne Helene Gelebart and his teammates investigated the cis-trans relaxation time of several azobenzene derivatives with varied end groups [15, 20-22]. As figure

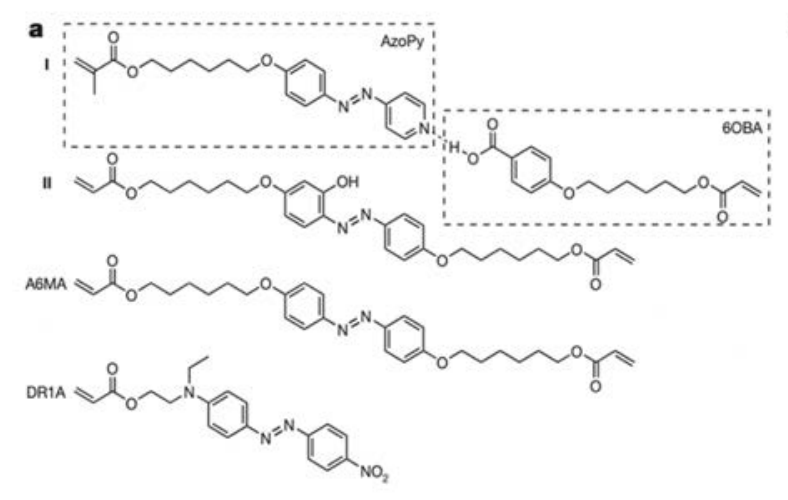

7 shows, the relaxation half-time of A6M6 is pretty long, meanwhile, azo-derivatives I and II shows faster relaxation. And the push-pull effect provided by hydrogen bond also play an important part in affecting the half-time [2].

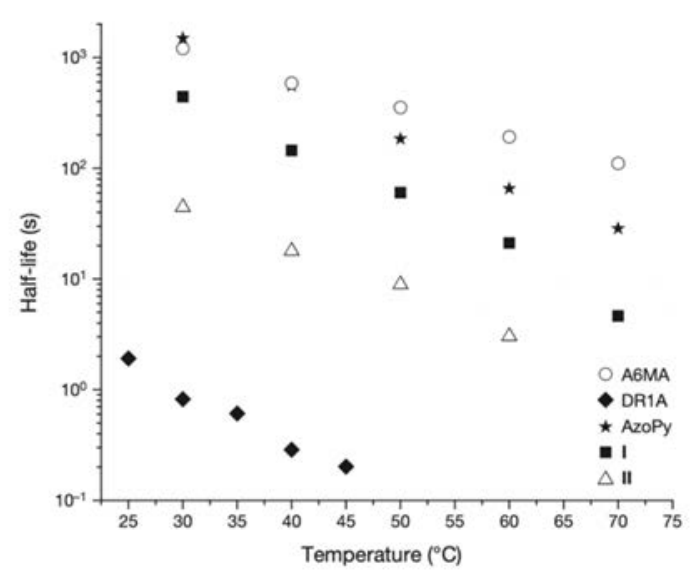

Fig. 7. Azo dyes and their cis-to-trans relaxation. a) Chemical structures of azo-derivatives. b) The half-lives of the azo-derivatives composed liquid-crystal cis-to-trans relaxation. Reproduced with permission from Ref. [20]

The experimental data explored by Lee and his team showed that the cantilever PD-20CL5 exist instantaneous and equilibrium bending at temperature above the $\mathrm{Tg}$. Upon the UV irradiation, the cantilever would bend instantaneously in a larger degree and achieve equilibrium status after a few seconds [29]. The time to reach equilibrium is strongly related to temperature. As the temperature goes up, the equilibrium time is longer; otherwise, the time needed to reach equilibrium state is shorter.

\section{Bending angle}


Kyung Min Lee and his team found out that the bending degree is largely dictated by the cross-link density. They tested thermomechanical property of different PD-20CL$\mathrm{xx}$ samples (curing time presented as $\mathrm{xx}$ ) like storage modulus $\left(\mathrm{E}^{\prime}\right)$, loss modulus ( $\left.\mathrm{E}^{\prime \prime}\right)$ and glass transition temperature (Tg) and calculated the cross-linked density by eq : $[23,24]$ (shown in figure $8 a$ ).

$$
v_{\mathrm{e}}=E_{\text {high }}^{\prime} /\left(3 R T_{\text {high }}\right)
$$

As figure 8c reveals, the bending angles of azo-LCN cantilever composed by PD-20CL-5 and PD-20CL-60 are different within the same polarization angle (the test principle of bending is shown in figure $8 \mathrm{~b}$ ). In fact, the bending angle is reduced when the cross-link density becomes larger.

What's more, they found out that the instant bending degree of azo-LCN cantilever contained PD-20CL-5 is under the influence of temperature (when the temperature is higher than the Tg). When the temperature rose, the instant bending angle becomes larger accordingly. (a)

\begin{tabular}{lcccc}
\hline sample code & $T_{\mathrm{g}}{ }^{a}\left({ }^{\circ} \mathrm{C}\right)$ & $T_{\text {high }}{ }^{b}(\mathrm{~K})$ & $E_{\text {high }}{ }^{c}(\mathrm{MPa})$ & $v_{\mathrm{e}}\left(\mathrm{mol} / \mathrm{dm}^{3}\right)$ \\
\hline PD-20CL-1 & 44 & 367 & 23.08 & 2.09 \\
PD-20CL-5 & 48 & 371 & 26.72 & 2.26 \\
PD-20CL-15 & 61 & 384 & 31.5 & 3.29 \\
PD-20CL-30 & 69 & 392 & 41.9 & 4.32 \\
PD-20CL-60 & 80 & 403 & 73.9 & 7.25 \\
PD-20CL-120 & 80 & 403 & 74.0 & 7.26
\end{tabular}

(b)

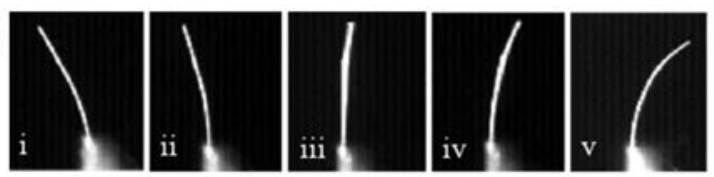

(c)

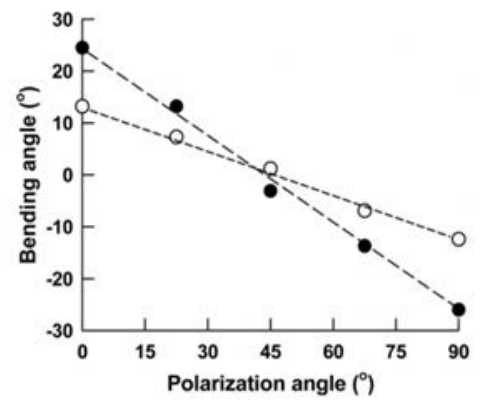

(d)

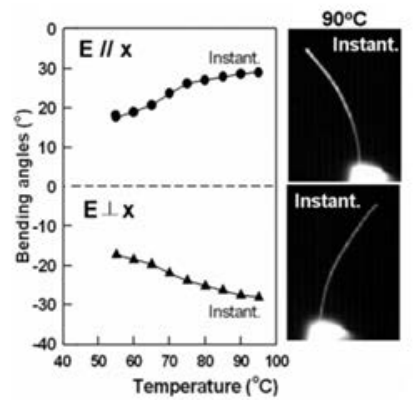

Fig. 8. a) The azo-LCN sample nomenclature of Tg, Thigh, $\mathrm{E}^{\prime}$ high, and crosslink density. b) Photoinduced bending of azo-LCN cantilever (originally vertical) containing PD-20CL-5 under irradiation of 442nm laser light in different polarization angles. (i) $0^{\circ}$, (ii) $20^{\circ}$, (iii) $45^{\circ}$, (iv) $70^{\circ}$, and (v) $90^{\circ}$ c) Summarization of accurate bending data through investigation on azo-LCN cantilever composed of PD-20CL-5 $(\bigcirc)$ and PD-20CL-60 ( ) when exposed to $80 \mathrm{~mW} / \mathrm{cm} 2$ of a $442 \mathrm{~nm}$ laser. d) Instant bending angles of azo-LCN cantilever contained PD-20CL5 under polarized laser in $\mathrm{E} \perp \mathrm{x}$ and $\mathrm{E} / / \mathrm{x}$ with various temperatures above Tg. Reproduced with permission from Ref. [12]

Iamsaard and his team detected that bending degree also influenced by the angle $\boldsymbol{\alpha}$ between twist-nematic molecular orientation which in the identical direction with the molecules at mid-plane of the film and the long axis of the ribbon. They cut the liquid crystal film containing azo-LCN and chiral dopants S-811 and R-811 [6] from various directions, and got the ribbon bending in different directions with diverse angle $\boldsymbol{\alpha}$. To illustrate the affected experimental law of $\boldsymbol{\alpha}$, figure 9a plots the photoacuation modes of azo-LCN cantilever doped with S-811 at different $\boldsymbol{\alpha}$. In the images shown in figure 9b, the direction of ribbon is from flat to left-hand spring, ring and right-hand spring at last, and bending degree here is like a spring which completely different from last mentioned ones. 

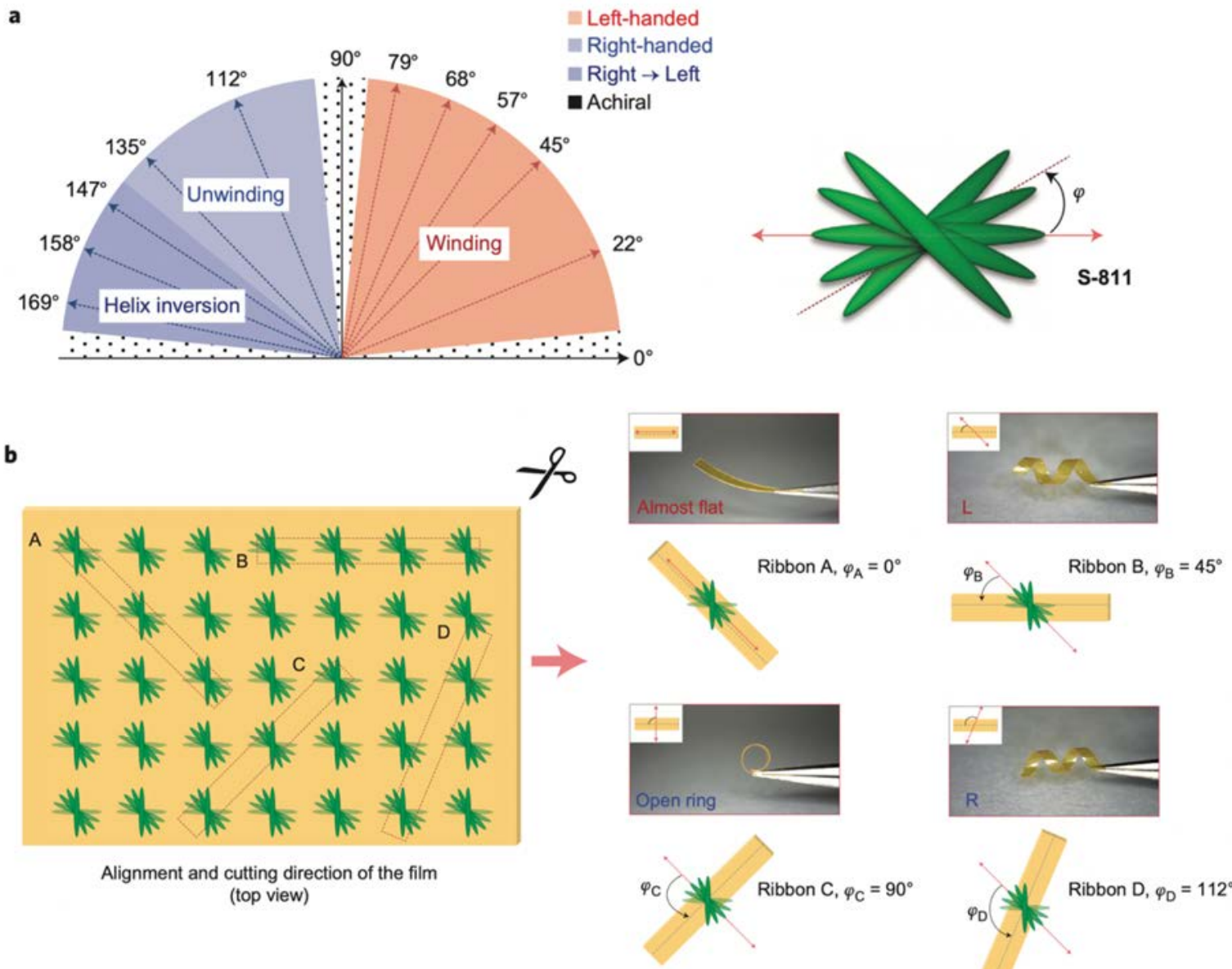

Fig. 9. a) Photoactuation modes of azo-LCN springs doped with S-811 twist left-hand directly. b) The ribbons bend in a variety of directions depend on the way they were cut.

\section{Conclusion and outlook}

Thereto, the photoinduced bending behaviors are usually described with properties such as bending direction, oscillating frequency and bending degree. Therefore, we summarized the influenced factors of the three increasingly interested properties. First, the phase-state of azobenzene, initial alignment of mesogens, the position of the crosslinked Azobenzene-containing liquidcrystalline polymers and polarization direction of light would affect the bending direction of AZ-LCP films. Then, we found out the factors contributing to oscillating frequency, including laser blocking frequency, cantilever thickness, materials, order parameter and substituents of azobenzene moiety. Later on, we pointed out the affecting factors of bending degree, including thermomechanical properties, temperature and the angle between molecules and long axis of ribbon.

Based on the operational analysis and detailed examination, the photo-responsive actuators have a lot of intriguing abilities, which includes no contact, remotely controlled and multiple adjustability. There are some research directions up to date with the rapid development of artificial intelligence (AI) such as highly integrated systems which can convert the external stimuli signal into chemical or physical changes and an electric powerdriven robot who can complete the expected missions. And there are also some processing problems, we can solve them by making the polymer actuators much softer or lighter, enabling them easily made into various shapes and have better mechanical performance with less fabrication process. The properties of AZ-containing LCP materials could also be further improved, we can boost the photo-responsive speed, increase the bending degree with versatile directionality control.

\section{References}

1. White, T. J.; Tabiryan, N. V.; Serak, S. V.; Hrozhyk, U. A.; Tondiglia, V. P.; Koerner, H.; Vaia, R. A.; Bunning, T. J., A high frequency photodriven polymer oscillator. Soft Matter 2008, 4 (9), 17961798.

2. Gelebart, A. H.; Mulder, D. J.; Varga, M.; Konya, A.; Vantomme, G.; Meijer, E.; Selinger, R. L.; Broer, D. J., Making waves in a photoactive polymer film. Nature 2017, 546 (7660), 632.

3. Wani, O. M.; Zeng, H.; Priimagi, A., A light-driven artificial flytrap. Nature communications 2017, 8, 15546. 
4. Yu, H., Recent advances in photoresponsive liquidcrystalline polymers containing azobenzene chromophores. Journal of Materials Chemistry C 2014, 2 (17), 3047-3054.

5. Tanchak, O. M.; Barrett, C. J., Light-induced reversible volume changes in thin films of azo polymers: The photomechanical effect. Macromolecules 2005, 38 (25), 10566-10570.

6. Iamsaard, S.; Aßhoff, S. J.; Matt, B.; Kudernac, T.; Cornelissen, J. J.; Fletcher, S. P.; Katsonis, N., Conversion of light into macroscopic helical motion. Nature chemistry 2014, 6 (3), 229-235.

7. Hu, J.; Wang, W.; Yu, H., Endowing Soft PhotoActuators with Intelligence. Advanced Intelligent Systems 2019, 1900050.

8. Yu, H.; Ikeda, T., Photocontrollable LiquidCrystalline Actuators. Advanced Materials 2011, 23 (19), 2149-2180.

9. White, T. J.; Broer, D. J., Programmable and adaptive mechanics with liquid crystal polymer networks and elastomers. Nature materials 2015, 14 (11), 1087-1098.

10. Yu, H., Photoresponsive liquid crystalline block copolymers: From photonics to nanotechnology. Progress in polymer science 2014, 39 (4), 781-815.

11. Priimagi, A.; Shimamura, A.; Kondo, M.; Hiraoka, T.; Kubo, S.; Mamiya, J.-I.; Kinoshita, M.; Ikeda, T.; Shishido, A., Location of the Azobenzene Moieties within the Cross-Linked Liquid-Crystalline Polymers Can Dictate the Direction of Photoinduced Bending. Acs Macro Letters 2012, 1 (1), 96-99.

12. Ikeda, T.; Nakano, M.; Yu, Y. L.; Tsutsumi, O.; Kanazawa, A., Anisotropic bending and unbending behavior of azobenzene liquid-crystalline gels by light exposure. Advanced Materials 2003, 15 (3), 201-+.

13. Kondo, M.; Yu, Y. L.; Ikeda, T., How does the initial alignment of mesogens affect the photoinduced bending behavior of liquid-crystalline elastomers? Angewandte Chemie-International Edition 2006, 45 (9), 1378-1382.

14. Bisoyi, H. K.; Li, Q., Light -Driven Liquid Crystalline Materials: From Photo -Induced Phase Transitions and Property Modulations to Applications. Chemical Reviews 2016, 116 (24), 15089-15166.

15. Bandara, H. D.; Burdette, S. C., Photoisomerization in different classes of azobenzene. Chemical Society Reviews 2012, 41 (5), 1809-1825.

16. Finkelmann, H.; Nishikawa, E.; Pereira, G. G.; Warner, M., A new opto-mechanical effect in solids. Physical Review Letters 2001, 87 (1).

17. Li, M. H.; Keller, P.; Li, B.; Wang, X. G.; Brunet, M., Light-driven side-on nematic elastomer actuators. Advanced Materials 2003, 15 (7-8), 569-572.

18. Yu, Y. L.; Nakano, M.; Ikeda, T., Directed bending of a polymer film by light - Miniaturizing a simple photomechanical system could expand its range of applications. Nature 2003, 425 (6954), 145-145.

19. Yu, H.; Dong, C.; Zhou, W.; Kobayashi, T.; Yang, H., Wrinkled liquid-crystalline microparticleenhanced photoresponse of PDLC-like films by coupling with mechanical stretching. Small 2011, 7 (21), 3039-3045.

20. García-Amorós, J.; Velasco, D., Recent advances towards azobenzene-based light-driven real-time information-transmitting materials. Beilstein journal of organic chemistry 2012, 8 (1), 1003-1017.

21. Van Oosten, C. L.; Bastiaansen, C. W.; Broer, D. J., Printed artificial cilia from liquid-crystal network actuators modularly driven by light. Nature materials 2009, 8 (8), 677-682.

22. Camacho-Lopez, M.; Finkelmann, H.; PalffyMuhoray, P.; Shelley, M., Fast liquid-crystal elastomer swims into the dark. Nature materials 2004, 3 (5), 307-310.

23. Cviklinski, J.; Tajbakhsh, A. R.; Terentjev, E. M., UV isomerisation in nematic elastomers as a route to photo-mechanical transducer. Eur. Phys. J. E 2002, 9 (5), 427-434.

24. Hogan, P. M.; Tajbakhsh, A. R.; Terentjev, E. M., uv manipulation of order and macroscopic shape in nematic elastomers. Phys. Rev. E 2002, 65 (4), 10.

25. Bandara, H. M. D.; Burdette, S. C., Photoisomerization in different classes of azobenzene. Chemical Society Reviews 2012, 41 (5), 1809-1825.

26. Cheng, Z. X.; Ma, S. D.; Zhang, Y. H.; Huang, S.; Chen, Y. X.; Yu, H. F., Photomechanical Motion of Liquid-Crystalline Fibers Bending Away from a Light Source. Macromolecules 2017, 50 (21), 8317 8324.

27. Yamada, M.; Kondo, M.; Mamiya, J.-i.; Yu, Y.; Kinoshita, M.; Barrett, C. J.; Ikeda, T., Photomobile polymer materials: Towards light-driven plastic motors. Angewandte Chemie-International Edition 2008, 47 (27), 4986-4988.

28. Yamada, M.; Kondo, M.; Miyasato, R.; Naka, Y.; Mamiya, J.-i.; Kinoshita, M.; Shishido, A.; Yu, Y.; Barrett, C. J.; Ikeda, T., Photomobile polymer materials-various three-dimensional movements. Journal of Materials Chemistry 2009, 19 (1), 60-62.

29. Lee, K. M.; Koerner, H.; Vaia, R. A.; Bunning, T. J.; White, T. J., Relationship between the Photomechanical Response and the Thermomechanical Properties of Azobenzene Liquid Crystalline Polymer Networks. Macromolecules 2010, 43 (19), 8185-8190. 